\title{
DIMENSION OF CERTAIN KERNEL SPACES OF LINEAR OPERATORS
}

\author{
ZUOWEI SHEN
}

(Communicated by J. Marshall Ash)

\begin{abstract}
Let $G$ be a semigroup of linear operators on a vector space $S$ into itself with the operation of composition. A subset of $G$ may be associated with a matroid $X$. We discuss the dimension of the kernels of certain linear operators induced in a natural way by the matroid structure on $X$.
\end{abstract}

\section{INTRODUCTION}

Let $X$ be an $s \times n$ matrix with columns $x \in \mathbb{R}^{s} \backslash\{0\}$ and rank $s$, and $\mathscr{B}(X)$ be the collection of bases for $\mathbb{R}^{s}$ consisting of column vectors of $X$. We consider the semigroup of commuting linear operators on $S=C^{\infty}\left(\mathbf{C}^{S}\right)$ defined by

$$
G_{1}:=\{q(D): q(x) \text { is a homogeneous polynomial }\}
$$

where $D=\left(\partial / \partial x_{1}, \ldots, \partial / \partial x_{s}\right)$.

For each $\xi \in X$, we associate an operator $q_{\xi}(D)$ to form a set $Q_{X} \subset G_{1}$. For each set of columns $V \subseteq X$, a partial differential operator $P_{V}$ is defined by

$$
P_{V}(D)=\prod_{\xi \in V} q_{\xi}(D) .
$$

Of particular interest are the intersection of kernels for the differential operators $P_{V}(D)$ with some restriction

$$
D\left(Q_{X}\right):=\left\{f \in C^{\infty}\left(\mathbb{R}^{s}\right): \prod_{y \in V} q_{y}(D) f=0, \forall \operatorname{dim}(X \backslash V)<s\right\} .
$$

Dahmen and Micchelli studied extensively kernels of this type and among other things gave the following theorem [DM3].

(1.1) Theorem. If $\operatorname{dim} D\left(Q_{B}\right)<\infty$ for all $B \in \mathscr{B}(X)$, then

$$
\operatorname{dim} D\left(Q_{X}\right) \leq \sum_{B \in \mathscr{B}(X)} \prod_{b \in B} \operatorname{deg} q_{b} .
$$

Received by the editors March 13, 1989 and, in revised form, August 7, 1990.

1980 Mathematics Subject Classification (1985 Revision). Primary 35G05, 39A70, 41A63.

Key words and phrases. Dimensin of null spaces, matroid structure.

Research supported in part by NSERC Canada under Grant \#A7687. 
Dahmen and Micchelli noted that equality holds in Theorem 1.1 in $\mathbb{R}^{2}$ and conjectured that the equality holds in general. Reading their paper [DM3] initiated our study of this problem, and a proof of the full conjecture is given in $\S 4$. We thank them for making a preprint of their paper available to us.

As Dahmen and Micchelli did in [DM2], we start with a somewhat more general setting. Let $X$ be a matroid with $\operatorname{rank}(X)=s$ and let $\left\{l_{x}: x \in X\right\}$ be a family of commuting linear operators with common domain and range on a vector space $S$. A corresponding kernel space is formed as above. Dahmen and Micchelli gave an upper bound for the dimension of this kernel space [DM2, Theorem 3.1]. They also gave a sufficient condition under which the dimension of the kernel space is equal to their upper bound [DM2, Theorem 3.3]. In this paper, we choose $\left\{l_{x}: x \in X\right\}$ from a commuting semigroup $G$ of linear operators mapping the vector space $S$ into itself. We prove that the dimension of the kernel space is equal to their upper bound if and only if the semigroup $G$ satisfies so-called " $s$-dimensionally additive" property.

The paper is organized as follows. In $\S 2$, we first give the basic concepts and our notation, and then state the main result of this paper. The key step in the proof of our main theorem is given in $\S 3$. Finally, we will affirm the conjecture in $\S 4$.

\section{Definitions AND MAIN RESUlts}

Let $X$ be an arbitrary set with finite cardinality $|X|=n$. A matroid structure on $X$ is defined by a collection of $\mathscr{T}$ of subsets of $X$ satisfying:

(i) The empty set is in $\mathscr{T}$.

(ii) If $V \in \mathscr{T}$, then any subset of $V$ is in $\mathscr{T}$.

(iii) For arbitrary $U, V \in \mathscr{T}$ with $|U|=|V|+1$, there exists $x \in U \backslash V$ such that $V \cup\{x\} \in \mathscr{T}$.

Such a collection $\mathscr{T}$ is said to be a collection of independent subsets of $X$. Any subset of $X$ that does not belong to $\mathscr{T}$ is called a dependent set. The set $X$ with matroid structure is called a matroid $X$.

Let $\varrho: 2^{X} \mapsto \mathbb{Z}_{+}$be defined on subsets $V \subset X$ by

$$
\varrho(V):=\max \{|Y|: Y \subset V, Y \in \mathscr{T}\} .
$$

Any element $y \in X$ with $\varrho(V \cup\{y\})=\varrho(V)$ is said to be dependent on $V$. An operator $\sigma: 2^{X} \mapsto 2^{X}$ defined by

$$
\sigma(V):=\bigcup\{Y \subset X: V \subset Y, \varrho(Y)=\varrho(V)\} \quad \forall V \subset X,
$$

maps $V$ to the set of all elements in $X$ that are dependent on $V$. The notation $\sigma_{X}$ is used whenever necessary to emphasize that $\sigma$ is defined relative to $X$.

A set $B \subset X$ is called a base for the matroid $X$ if $B$ is a maximal independent subset of $X$. It can be proved that every base of $X$ has the same cardinality, $\varrho(X)$, which is called the rank of $X$. This paper will deal mainly with matroids of a given rank $s$. The collection $\mathscr{B}(X)$ of all bases is described 
as

$$
\mathscr{B}(X):=\{B \subset X:|B|=\varrho(B)=\varrho(X)\} .
$$

We also need to distinguish the collection of all subsets of $X$ whose complements are not of full rank

$$
\mathscr{A}(X):=\{V \subset X: \varrho(X \backslash V)<\varrho(X)\},
$$

and the collection of "hyperplanes" in $X$,

$$
\mathscr{H}(X):=\{H \subset X: \sigma(H)=H, \varrho(H)=\varrho(X)-1\} .
$$

For a matroid $X$ and a subset $Y$ of $X$, the restriction of the matroid structure to $Y$ forms a matroid on $Y$ with this structure $Y$ is called a submatroid of $X$. For an arbitrary subset $Y$ of a matroid $X$, we will always assume that the submatroid structure is imposed.

Let $G$ denote a semigroup formed from commuting linear operators that map a vector space $S$ to itself. The group operation is taken as composition of linear operators. Only such semigroups are considered in this paper, and for convenience, we refer to them as the semigroup $G$ or simply $G$.

We associate linear operators from the semigroup $G$ to elements of $X$, $\xi \mapsto l_{\xi}$, in an arbitrary fashion. For each subset $V \subset X, L_{V}$ is the associated set of linear operators from $G$;

$$
L_{V}:=\left\{l_{\xi}: \xi \in V\right\} .
$$

The operators in $L_{V}$ are combined under the semigroup operation to give a linear operator $P_{V}$ corresponding to $V \subset X$;

$$
P_{V}:=\prod_{\xi \in V} l_{\xi} .
$$

These operators are well defined since the operators are commutative.

Next we describe the kernels of the linear operators of interest to us. For any matroid $X$ with a set of associated linear operators $L_{X}$, the space $K\left(L_{X}\right)$ is defined as

$$
K\left(L_{X}\right):=\left\{f \in S: P_{V} f=0, \forall V \in \mathscr{A}(X)\right\},
$$

which is equivalently described as

$$
K\left(L_{X}\right):=\left\{f \in S: P_{X \backslash H} f=0, \forall H \in \mathscr{H}(X)\right\} .
$$

In particular, this definition applies to any submatroid $Y$ with the corresponding $L_{Y} \subset L_{X}$. For example, if $Y=B, B \in \mathscr{B}(X)$, then the kernel has the simplified form

$$
K\left(L_{B}\right)=\bigcap_{b \in B}\left\{f \in S: l_{b} f=0\right\} .
$$

We say that a set of linear operators is well associated with the matroid $Y$ if $\varrho(Y)=s$ and for each $B \in \mathscr{B}(Y), \operatorname{dim} K\left(L_{B}\right)<\infty$. 
Dahmen and Micchelli have proved that

$$
\operatorname{dim} K\left(L_{X}\right) \leq \sum_{B \in \mathscr{B}(X)} \operatorname{dim} K\left(L_{B}\right)
$$

in [DM2, Theorem 3.1]. We want to find conditions on $G$ under which the equality holds for any choice $L_{X} \subset G$ of operators associated with $X$. For this purpose, we say that a semigroup $G$ is excellently associated with the matroid $X$ if whenever a collection of operators $L_{X} \subset G$ is associated with $X$,

$$
\operatorname{dim} K\left(L_{X}\right)=\sum_{B \in \mathscr{B}(X)} \operatorname{dim} K\left(L_{B}\right) .
$$

As we shall see, this property of $G$ is related to another concept that we call the $s$-dimensional additivity of the semigroup $G$. Let

$$
F_{1}=\left\{l_{1}, \ldots, l_{j}, \ldots, l_{s}\right\} \text { and } F_{2}=\left\{l_{1}, \ldots, \tilde{l}_{j}, \ldots, l_{s}\right\}
$$

be two subsets of linear operators in $G$, and form a new subset $F$ from them by

$$
F:=\left\{l_{1}, \ldots, \tilde{l}_{j} l_{j}, \ldots, l_{s}\right\},
$$

then the kernel spaces corresponding to $F_{1}, F_{2}$, and $F$ are respectively

$$
K\left(F_{1}\right)=\bigcap_{i=1}^{s} \operatorname{ker} l_{i}, \quad K\left(F_{2}\right)=\operatorname{ker} \tilde{l}_{j} \cap\left(\bigcap_{\substack{i=1 \\ i \neq j}}^{s} \operatorname{ker} l_{i}\right),
$$

and

$$
K(F)=\operatorname{ker}\left(\tilde{l}_{j} l_{j}\right) \cap\left(\bigcap_{\substack{i=1 \\ i \neq j}}^{s} \operatorname{ker} l_{i}\right),
$$

where $\operatorname{ker} l:=\{f \in S: l f=0\}$. We say that $G$ is s-dimensionally additive if the following condition holds for arbitrary $F_{1}, F_{2}$, and $F$ as above:

$$
\operatorname{dim} K(F)=\operatorname{dim} K\left(F_{1}\right)+\operatorname{dim} K\left(F_{2}\right) .
$$

Since our proofs are based on an induction argument, we shall often consider matroids of the form $X \cup \zeta$ with the submatroid $X$ of the same rank, $\varrho(X)=$ $\varrho(X \cup \zeta)$. In such a situation we consider the relations between $\mathscr{H}(X \cup \zeta)$ and $\mathscr{H}(X)$. In particular, a special role is played by the subcollection

$$
\mathscr{H}_{\zeta}(X):=\left\{H \in \mathscr{H}(X): \sigma_{X \cup \zeta}(H)=H\right\}=\mathscr{H}(X \cup \zeta) \cap \mathscr{H}(X) .
$$

The following theorem is very important to the proof of our main result. Its proof will be the topic of the next section.

(2.2) Theorem. Let $G$ be an s-dimensionally additive semigroup, and suppose that $G$ is excellently associated with any matroid $X^{\prime}$ satisfying $\left|X^{\prime}\right| \leq|X|$ and $\varrho\left(X^{\prime}\right)=s$. For any matroid of the form $X \cup \zeta$ with $\varrho(X \cup \zeta)=\varrho(X)=s$ and 
any $H_{0} \in \mathscr{H}_{\zeta}(X)$, if $L_{X \cup \zeta} \subset G$ is associated with $X \cup \zeta$ and $L_{X}$ and $L_{H_{0} \cup \zeta}$ are well associated with $X$ and $H_{0} \cup \zeta$ respectively, then the system

$$
\begin{aligned}
& P_{X \backslash H_{0}} f=\psi, \\
& P_{X \backslash H} f=0 \quad \forall H \in \mathscr{H}(X) \backslash\left\{H_{0}\right\}
\end{aligned}
$$

is solvable for any $\psi \in K\left(L_{H_{0} \cup \zeta}\right)$.

The usefulness of the solvability of (2.3) was recognized already in [DM2]. Using Theorem (2.2), we can prove our main result.

(2.4) Theorem. The semigroup $G$ is s-dimensionally additive if and only if $G$ is excellently associated with any matroid $X$ of rank $\varrho(X)=s$, i.e. for any $L_{X} \subset G$ associated with $X$,

$$
\operatorname{dim} K\left(L_{X}\right)=\sum_{B \in \mathscr{B}(X)} \operatorname{dim} K\left(L_{B}\right) .
$$

Proof. " $\Leftarrow$ " Suppose $F_{1}, F_{2}$, and $F$ are the sets of linear operators given as above. Let $X=\left\{e_{1}, \ldots, e_{j}, \ldots, e_{s}, \tilde{e}_{j}\right\}$ where $e_{1}, \ldots, e_{s}$ are linearly independent vectors in $\mathbb{R}^{s}$, and $\tilde{e}_{j}=e_{j}$. Then $X$ has a natural matroid structure with $\varrho(X)=s$. Moreover, $\mathscr{B}(X)=\left\{B_{1}, B_{2}\right\}$ with,

$$
B_{1}=\left\{e_{1}, \ldots, e_{j}, \ldots, e_{s}\right\}, \quad B_{2}=\left\{e_{1}, \ldots, \tilde{e}_{j}, \ldots, e_{s}\right\} .
$$

The set of linear operators $L_{X}:=\left\{l_{1}, \ldots, l_{j}, \ldots, l_{s}, \tilde{l}_{j}\right\}$ is associated to $X$ by the correspondence $e_{j} \leftrightarrow l_{j}, j=1, \ldots, s$ and $\tilde{e}_{j} \leftrightarrow \tilde{l}_{j}$. We observe that $K(F)=K\left(L_{X}\right)$. Therefore, since $G$ is excellently associated with $X$, we have

$$
\begin{aligned}
\operatorname{dim} K(F)=\operatorname{dim} K\left(L_{X}\right) & =\operatorname{dim} K\left(L_{B_{1}}\right)+\operatorname{dim} K\left(L_{B_{2}}\right) \\
& =\operatorname{dim} K\left(F_{1}\right)+\operatorname{dim} K\left(F_{2}\right) .
\end{aligned}
$$

Thus, $G$ is $s$-dimensionally additive.

" $\Rightarrow$ " For the case that $L_{X}$ is not well associated with $X$, the equality holds simply because there exists a $B \in \mathscr{B}(X)$ such that $\operatorname{dim} K\left(L_{B}\right)=\infty$ and $K\left(L_{B}\right) \subseteq K\left(L_{X}\right)$.

For the case that $L_{X}$ is well associated with $X$, we will prove the equality by induction on $|X|$. When $\varrho(X)=|X|=s$, the theorem is obviously true. Suppose now that the theorem holds for all $X^{\prime}$ with $\left|X^{\prime}\right| \leq|X|$ and $\varrho\left(X^{\prime}\right)=s$. We want to establish it for $X \cup \zeta$ with $\varrho(X \cup \zeta)=s$. We may assume that $\varrho(X)=s$, otherwise, we can relabel the matroid $X \cup \zeta$ so that this is the case.

The map $P_{X \backslash H} f$ maps $K\left(L_{X \cup \zeta}\right)$ into $K\left(L_{H \cup \zeta}\right)$ for each $H \in \mathscr{H}_{\zeta}(X)$. Indeed, since for arbitrary $V \in \mathscr{A}(H \cup \zeta)$ and $f \in K\left(L_{X \cup \zeta}\right)$

$$
\varrho(X \cup \zeta \backslash(V \cup(X \backslash H)))=\varrho((H \cup \zeta) \backslash V)<s,
$$

we have $P_{V} P_{X \backslash H} f=0$; i.e., $P_{X \backslash H} f \in K\left(L_{H \cup \zeta}\right)$ whenever $f \in K\left(L_{X \cup \zeta}\right)$. Therefore, we consider the operator

$$
\mathscr{P}: K\left(L_{X \cup \zeta}\right) \mapsto \prod_{H \in \mathscr{\mathscr { F } _ { \zeta }}(X)} K\left(L_{H \cup \zeta}\right),
$$


where $\prod_{H \in \mathscr{P}_{\zeta}(X)} K\left(L_{H \cup \zeta}\right)$ is the Cartesian product with component operators $P_{X \backslash H}, H \in \mathscr{\mathscr { L }}_{\zeta}(X)$. Observe that for $f \in K\left(L_{X \cup \zeta}\right)$,

$$
P_{X \backslash H} f=P_{X \cup \zeta \backslash \sigma_{X \cup \zeta}(H)} f=0 \quad \forall H \in \mathscr{H}(X) \backslash \mathscr{H}_{\zeta}(X),
$$

since $\zeta \in \sigma_{X \cup \zeta}(H)$ in this case. Hence $\operatorname{ker}(\mathscr{P})=K\left(L_{X}\right)$.

Therefore,

$$
\operatorname{dim} K\left(L_{X \cup \zeta}\right) \leq \operatorname{dim} K\left(L_{X}\right)+\sum_{H \in \mathscr{\ell _ { \zeta }}(X)} \operatorname{dim} K\left(L_{H \cup \zeta}\right) .
$$

But by the induction hypothesis,

$$
\begin{aligned}
\operatorname{dim} & K\left(L_{X}\right)+\sum_{H \in \mathscr{P}_{\zeta}(X)} \operatorname{dim} K\left(L_{H \cup \zeta}\right) \\
& =\sum_{B \in \mathscr{B}(X)} \operatorname{dim} K\left(L_{B}\right)+\sum_{H \in \mathscr{F}_{\zeta}(X)} \sum_{B \in \mathscr{B}(H \cup \zeta)} \operatorname{dim} K\left(L_{B}\right) \\
& =\sum_{B \in \mathscr{F}(X \cup \zeta)} \operatorname{dim} K\left(L_{B}\right) .
\end{aligned}
$$

Equality holds in (2.7) if and only if the mapping $\mathscr{P}$ is onto since the righthand side is finite. The latter is equivalent to the statement: For arbitrary $H_{0} \in \mathscr{H}_{\zeta}(X)$ and $\psi \in K\left(L_{H_{0} \cup \zeta}\right)$, the system of equations

$$
\begin{aligned}
P_{X \backslash H_{0}} f & =\psi, \\
P_{X \backslash H} f & =0 \quad \forall H \in \mathscr{H}_{\zeta}(X) \backslash\left\{H_{0}\right\}
\end{aligned}
$$

has a solution with $f \in K\left(L_{X \cup \zeta}\right)$. We claim that system (2.8) has a solution $f \in K\left(L_{X \cup \zeta}\right)$ if and only if system (2.3) has a solution in $S$.

Let $f \in K\left(L_{X \cup \zeta}\right)$ be a solution of system (2.8), then $f$ is a solution of system (2.3) by (2.6). On the other hand, if $f$ is a solution of (2.3), then obviously $f$ is a solution of system (2.8). Therefore, we only need to show that $f \in K\left(L_{X \cup \zeta}\right)$. But it can be easily proved by the following proposition:

(2.9) Proposition. Let $H_{0} \in \mathscr{H}_{\zeta}(X)$ be given. For any $H \in \mathscr{H}(X \cup \zeta) \backslash\left\{H_{0}\right\}$, there exists $\widetilde{H} \in \mathscr{H}(X) \backslash\left\{H_{0}\right\}$ such that $(X \cup \zeta) \backslash H \supset X \backslash \widetilde{H}$.

Proof. Let $H \in \mathscr{H}(X \cup \zeta) \backslash\left\{H_{0}\right\}$. If $H \backslash \zeta \in \mathscr{H}(X)$, then we take $\widetilde{H}=H \backslash \zeta$. If $H \backslash \zeta \notin \mathscr{H}(X)$, then we must have $\varrho(H \backslash \zeta)<s-1$. In this case, choose any $y^{*} \in X \backslash H_{0}$. Then for any hyperplane $\widetilde{H} \in \mathscr{H}(X)$ containing $(H \backslash \zeta) \cup y^{*}$, we have

$$
(X \cup \zeta) \backslash H=X \backslash(H \backslash \zeta) \supseteq X \backslash \widetilde{H} .
$$

\section{THE MAIN INDUCTION STEP}

In this section we prove Theorem 2.2 by induction on the number of elements of $X$. Assume that the conditions of the theorem hold and assume that the 
theorem holds for any matroid $\left|X^{\prime}\right| \leq|X|-1$. We establish it for the matroids of the form $X$ with $\varrho(X)=s=\varrho(X \cup \zeta)$.

Fix $H_{0} \in \mathscr{H}_{\zeta}(X)$. We consider the operator $P_{X \backslash H_{0}}$ restricted to the space defined by

$$
K_{\zeta, H_{0}}\left(L_{X \cup \zeta}\right):=\left\{f \in S: l_{\zeta} P_{X \backslash H_{0}} f=0 \text { and } P_{X \backslash H} f=0 \forall H \in \mathscr{H}(X) \backslash\left\{H_{0}\right\}\right\} .
$$

From Proposition 2.9, it is clear that

$$
K\left(L_{X}\right) \subseteq K_{\zeta, H_{0}}\left(L_{X \cup \zeta}\right) \subseteq K\left(L_{X \cup \zeta}\right) .
$$

In the proof of Theorem 2.4, it was shown that

$$
P_{X \backslash H_{0}}: K_{\zeta, H_{0}}\left(L_{X \cup \zeta}\right) \mapsto K\left(L_{H_{0} \cup \zeta}\right) .
$$

It is clear from the definitions that the mapping (3.3) has kernel $K\left(L_{X}\right)$. Therefore,

$$
\begin{aligned}
\operatorname{dim} K_{\zeta, H_{0}}\left(L_{X \cup \zeta}\right) & \leq \operatorname{dim} K\left(L_{X}\right)+\operatorname{dim} K\left(L_{H_{0} \cup \zeta}\right) \\
& =\sum_{B \in \mathscr{B}(X)} \operatorname{dim} K\left(L_{B}\right)+\sum_{B \in \mathscr{B}\left(H_{0} \cup \zeta\right)} \operatorname{dim} K\left(L_{B}\right) .
\end{aligned}
$$

The equality follows from the fact that $G$ is excellently associated with $H_{0} \cup \zeta$ and $X$ by hypothesis. Since the right-hand side is finite, equality in (3.4) is equivalent to the map (3.3) being onto $K\left(L_{H_{0} \cup \zeta}\right)$, and this in turn is equivalent to the solvability of the system (2.3).

We wish to solve the system (2.3) for a given $\varphi \in K\left(L_{H_{0} \cup \zeta}\right)$. We first associate another set of operators with $X$ in addition to the given operators $L_{X}$. We pick $x^{*} \in X$ with $x^{*} \notin H_{0}$ and let $X^{*}=X \backslash x^{*}$. The set of operators $\widetilde{L}_{X}=\widetilde{L}_{X^{*} \cup x^{*}}$ associated with $X$ is defined as follows

$$
\tilde{l}_{x}:= \begin{cases}l_{x}, & x \neq x^{*} \\ l_{x} l_{\zeta}, & x \neq x^{*}\end{cases}
$$

There are two cases. The first case includes the starting point for the induction, $|X|=s$. If $\varrho\left(X \backslash x^{*}\right)=s-1$, then for arbitrary $B \in \mathscr{B}(X), x^{*} \in B$ and all the other elements belong to $H_{0}$. Thus, if $B=\left\{x^{*}, x_{1}, \ldots, x_{s-1}\right\}$, then $B_{\zeta}:=\left\{\zeta, x_{1}, \ldots, x_{s-1}\right\} \in \mathscr{B}\left(H_{0} \cup \zeta\right)$ and from the $s$-dimensional additivity of $G$ and the fact that $L_{X}$ and $L_{H_{0} \cup \zeta}$ are well associated with $X$ and $H_{0} \cup \zeta$ respectively, we have

$$
\operatorname{dim} K\left(\widetilde{L}_{B}\right)=\operatorname{dim} K\left(L_{B}\right)+\operatorname{dim} K\left(L_{B_{\zeta}}\right)<\infty .
$$

This implies that $\widetilde{L}_{X}$ is well associated with $X$. Finally, since $G$ is excellently associated with $X$, we have

$$
\operatorname{dim} K\left(\widetilde{L}_{X}\right)=\sum_{B \in \mathscr{B}(X)} \operatorname{dim} K\left(\widetilde{L}_{B}\right)=\sum_{B \in \mathscr{B}(X)} \operatorname{dim} K\left(L_{B}\right)+\sum_{B \in \mathscr{B}\left(H_{0} \cup \zeta\right)} \operatorname{dim} K\left(L_{B}\right) .
$$


Since $K_{\zeta, H_{0}}\left(L_{X \cup \zeta}\right)=K\left(\widetilde{L}_{X}\right)$ in this case, equality holds in (3.4). Therefore, the system (2.3) is solvable for $H_{0}$.

The case $\varrho\left(X \backslash x^{*}\right)=s$ takes a lot more work. Clearly, $\left|X^{*}\right|<|X|$ and $\varrho\left(X^{*}\right)=s$. We first note that $H_{0} \in \mathscr{H}_{x^{*}}\left(X^{*}\right)$, because $H_{0} \in \mathscr{H}(X), x^{*} \notin$ $H_{0}$, and $X=X^{*} \cup x^{*}$. In order to apply the induction hypothesis to $X^{*}$, we need to verify that $\widetilde{L}_{X^{*}}$ and $\widetilde{L}_{H_{0} \cup x^{*}}$ are well associated with $X^{*}$ and $H_{0} \cup x^{*}$ respectively. The first is obvious since $\widetilde{L}_{X^{*}} \subset L_{X}$. For the second statement, if $B \in \mathscr{B}\left(H_{0} \cup x^{*}\right)$, then $B=\left\{x^{*}, x_{1}, \ldots, x_{s-1}\right\} \in \mathscr{B}(X)$ and $B_{\zeta}=\left\{\zeta, x_{1}, \ldots, x_{s-1}\right\} \in \mathscr{B}(X \cup \zeta)$ which was the case considered above.

From the relation (3.5), we have that for all $H \in \mathscr{H}\left(X^{*}\right)$,

$$
\widetilde{P}_{X^{*} \backslash H}:=\prod_{x \in X^{*} \backslash H} \tilde{l}_{x}=\prod_{x \in X^{*} \backslash H} l_{x}=P_{X^{*} \backslash H^{*}}
$$

Therefore, the kernel $K_{x^{*}, H_{0}}\left(\widetilde{L}_{X^{*} \cup x^{*}}\right)$ has the representation

$$
\begin{aligned}
K_{x^{*}, H_{0}}\left(\widetilde{L}_{X^{*} \cup x^{*}}\right)=\left\{f \in S: l_{x^{*}} l_{\zeta} P_{X^{*} \backslash H_{0}} f=0,\right. & \text { and } P_{X^{*} \backslash H} f=0, \\
& \left.\forall H \in \mathscr{H}\left(X^{*}\right) \backslash\left\{H_{0}\right\}\right\} .
\end{aligned}
$$

Since $\left|X^{*}\right|<|X|, \widetilde{L}_{X^{*}}$ and $\widetilde{L}_{H_{0} \cup x^{*}}$ are well associated with the matroid $X^{*}$, and $H_{0} \cup X^{*}$, respectively, the induction hypothesis yields the solvability of the system

$$
\begin{aligned}
& P_{X^{*} \backslash H_{0}} f=\psi \quad \psi \in K\left(\widetilde{L}_{H_{0} \cup x^{*}}\right), \\
& P_{X^{*} \backslash H} f=0 \quad \forall H \in \mathscr{H}\left(X^{*}\right) \backslash\left\{H_{0}\right\} .
\end{aligned}
$$

It is easy to verify that $l_{x^{*}}$ maps $K\left(\widetilde{L}_{H_{0} \cup x^{*}}\right)$ into $K\left(L_{H_{0} \cup \zeta}\right)$ with kernel $K\left(L_{H_{0} \cup x^{*}}\right)$. Since $\left|H_{0} \cup x^{*}\right| \leq|X|, G$ is excellently associated to $H_{0} \cup x^{*}$. That is,

$$
\begin{aligned}
\operatorname{dim} K\left(\widetilde{L}_{H_{0} \cup x^{*}}\right) & =\sum_{B \in \mathscr{B}\left(H_{0} \cup x^{*}\right)} \operatorname{dim} K\left(\widetilde{L}_{B}\right) \\
& =\sum_{B \in \mathscr{B}\left(H_{0} \cup x^{*}\right)} \operatorname{dim} K\left(L_{B}\right)+\sum_{B \in \mathscr{B}\left(H_{0} \cup \zeta\right)} \operatorname{dim} K\left(L_{B}\right) \\
& =\operatorname{dim} K\left(L_{H_{0} \cup x^{*}}\right)+\operatorname{dim} K\left(L_{H_{0} \cup \zeta}\right) .
\end{aligned}
$$

Hence, $l_{x^{*}}$ maps $K\left(\widetilde{L}_{H_{0} \cup x^{*}}\right)$ onto $K\left(L_{H_{0} \cup \zeta}\right)$ since the dimension of $K\left(\widetilde{L}_{H_{0} \cup x^{*}}\right)$ is finite. Thus, for any given $\varphi \in K\left(L_{H_{0} \cup \zeta}\right)$, we can find $\psi \in K\left(\widetilde{L}_{H_{0} \cup x^{*}}\right)$ such that $l_{x^{*}} \psi=\varphi$.

Let $f$ be a solution of (3.7). Since $l_{x^{*}} \psi=\varphi$, we have

$$
P_{X \backslash H_{0}} f=l_{x^{*}} P_{X^{*} \backslash H_{0}} f=l_{x^{*}} \psi=\varphi .
$$


For $H \in \mathscr{H}(X) \backslash\left\{H_{0}\right\}$, by Proposition 2.9, there exists $\widetilde{H} \in \mathscr{H}\left(X^{*}\right) \backslash\left\{H_{0}\right\}$ such that $(X) \backslash H \supseteq X^{*} \backslash \widetilde{H}$, hence $P_{X \backslash H} f=P_{(\cdots)} P_{X^{*} \backslash \widetilde{H}} f=0$. This shows that $f$ is a solution of $(2.3)$.

\section{The CONJeCture of Dahmen AND Micchelli}

Let $X$ be an $s \times n$ matrix with columns $\xi \in \mathbb{R}^{s} \backslash\{0\}$ and rank $s$. Let $\mathscr{T}$ be the collection of all subsets of linearly independent column vectors of $X$. Then $X$ with $\mathscr{T}$ form a natural matroid structure with $\operatorname{rank} \varrho(X)=s$. For $S=C^{\infty}\left(\mathbf{C}^{s}\right)$, we consider the semigroup of commuting linear operators on $S$ defined by

$$
G_{1}:=\{q(D): q(x) \text { is a homogeneous polynomial }\}
$$

where $D=\left(\partial / \partial x_{1}, \ldots, \partial / \partial x_{s}\right)$.

Given $\left\{q_{1}(D), \ldots, q_{s}(D)\right\}$ in $G_{1}$, we set

$$
\operatorname{ker}\left(q_{1}, \ldots, q_{s}\right):=\bigcap_{i=1}^{s} \operatorname{ker} q_{i}(D),
$$

where $\operatorname{ker} q_{i}(D):=\left\{f \in C^{\infty}\left(\mathbf{C}^{S}\right): q_{i}(D) f=0\right\}$.

Stiller [S] has established a formula for $\operatorname{dim} \operatorname{ker}\left(q_{1}, \ldots, q_{s}\right)$. Dahmen and Micchelli gave the formula in the following nice form [DM3, Corollary 2.4]:

$$
\operatorname{dim} \operatorname{ker}\left(q_{1}, \ldots, q_{s}\right)<\infty \Rightarrow \operatorname{dim} \operatorname{ker}\left(q_{1}, \ldots, q_{s}\right)=\prod_{i=1}^{s} \operatorname{deg} q_{i},
$$

which makes the proof of the $s$-dimensional additivity of $G_{1}$ easier.

Using these observations, it is easy to see that $G_{1}$ is $s$-dimensionally additive. Indeed, let $F_{1}=\left\{q_{1}, \ldots, q_{j}, \ldots, q_{s}\right\}, F_{2}=\left\{q_{1}, \ldots, \tilde{q}_{j}, \ldots, q_{s}\right\}$, and $F=$ $\left\{q_{1}, \ldots, \tilde{q}_{j} q_{j}, \ldots, q_{s}\right\}$. It is easily seen that $\operatorname{dim} \operatorname{ker}(F)<\infty$ if and only if both $\operatorname{dim} \operatorname{ker}\left(F_{1}\right)<\infty$ and $\operatorname{dim} \operatorname{ker}\left(F_{2}\right)<\infty$. Furthermore, $\operatorname{dim} \operatorname{ker}(F)<\infty$ implies

$$
\begin{aligned}
\operatorname{dim} \operatorname{ker}(F) & =\left(\prod_{\substack{i=1 \\
i \neq j}}^{s} \operatorname{deg} q_{i}\right)\left(q_{j}+\widetilde{q}_{j}\right) \\
& =\operatorname{dim} \operatorname{ker}\left(F_{1}\right)+\operatorname{dim} \operatorname{ker}\left(F_{2}\right)
\end{aligned}
$$

by (4.1). Therefore, $G_{1}$ is $s$-dimensionally additive.

Let $Q_{X}$ be a set of linear operators in $G_{1}$ associated with $X$. The corresponding kernel space, as defined in $\S 2$, can be described as

$$
D\left(Q_{X}\right):=\left\{f \in C^{\infty}\left(\mathbf{C}^{s}\right): \prod_{y \in V} q_{y}(D) f=0, \forall V \in \mathscr{A}(X)\right\} .
$$

The following theorem affirms a conjecture stated by Dahmen and Micchelli in [DM3]. 
(4.2) Theorem. We have

$$
\operatorname{dim} D\left(Q_{X}\right)=\sum_{B \in \mathscr{B}(X)} \operatorname{dim} D\left(Q_{B}\right) .
$$

Moreover, if $\operatorname{dim} D\left(Q_{B}\right)<\infty$ for all $B \in \mathscr{B}(X)$, then

$$
\operatorname{dim} D\left(Q_{X}\right)=\sum_{B \in \mathscr{B}(X)} \prod_{b \in B} \operatorname{deg} q_{b} .
$$

Proof. Since $G_{1}$ is $s$-dimensionally additive, Theorem 2.4 shows that $G_{1}$ is excellently associated with $X$; i.e., the first equality holds. Then, the second equality holds by (4.1).

\section{ACKNOWLEDGMENT}

The author wishes to express his gratitude to his supervisor, Professor S. D. Riemanschneider, and his teacher, Professor R. Q. Jia, for their patience and for being a source of encouragement and guidance.

\section{REFERENCES}

[BR] C. de Boor and A. Ron, On polynomial ideals of finite co-dimension with application to box splines theory, J. Math. Anal. Appl. (1988) (to appear).

[DM1] W. Dahmen and C. A. Micchelli, On the local linear independence of translates of a box spline, Studia Math. 82 (1985), 243-263.

[DM2] _ On multivariate E-splines, Adv. in Math. 76 (1989), 33-93.

[DM3] __ Local dimension of piecewise polynomial space syzygies and solutions of systems of partial equations, I.B.M. Research Report, Math. Nachr. 1988 (to appear).

[S] P. Stiller, Vector bundles on complex projective spaces and systems of partial differential equations. I, Trans. Amer. Math. Soc. 298 (1986), 537-548.

Department of Mathematics, University of Alberta, Edmonton, Alberta T6G 2G1 Canada 